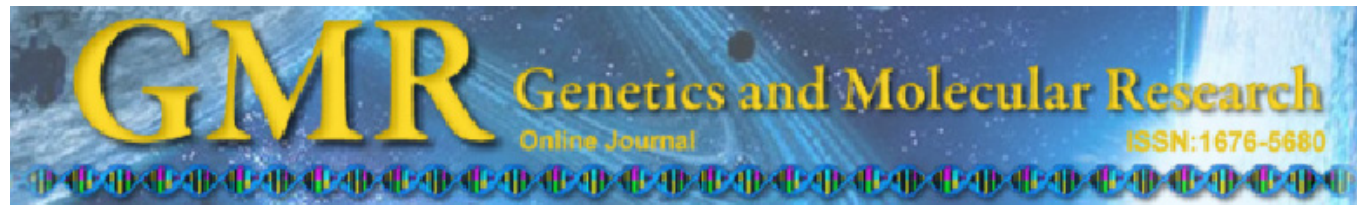

\title{
Association between matrix metalloproteinase-3 polymorphism and anterior cruciate ligament ruptures
}

S. Malila ${ }^{1,2}$, P. Yuktanandana ${ }^{3}$, S. Saowaprut ${ }^{4}$, W. Jiamjarasrangsi $i^{5}$ and S. Honsawek ${ }^{1}$

${ }^{1}$ Department of Biochemistry, Faculty of Medicine, Chulalongkorn University, Bangkok, Thailand

${ }^{2}$ Graduate Program in Medical Sciences, Faculty of Medicine, Chulalongkorn University, Bangkok, Thailand

${ }^{3}$ Department of Orthopaedics, Faculty of Medicine, Chulalongkorn University, Bangkok, Thailand

${ }^{4}$ Institute of Orthopaedics, Lerdsin Hospital, Bangkok, Thailand

${ }^{5}$ Department of Preventive and Social Medicine,

Faculty of Medicine, Chulalongkorn University, Bangkok, Thailand

Corresponding author: S. Honsawek

E-mail: Sittisak.H@chula.ac.th

Genet. Mol. Res. 10 (4): 4158-4165 (2011)

Received October 10, 2010

Accepted June 26, 2011

Published October 31, 2011

DOI http://dx.doi.org/10.4238/2011.October.31.1

ABSTRACT. Anterior cruciate ligament (ACL) ruptures are considered to be the most severe joint injury in sports. However, the precise etiologies of ACL injuries are not fully understood. Recently, the gene encoding the matrix metalloproteinase-3 (MMP-3, stromelysin-1) was shown to be associated with anterior cruciate ligament ruptures. The $5 \mathrm{~A} / 6 \mathrm{~A}$ polymorphism in the promoter of the MMP-3 gene affects the regulation of MMP-3 gene expression. We examined the association between polymorphism within -1612 of the MMP-3 gene and ACL rupture in an independent population. Eighty-six participants between 20 and 40 years of age with surgically diagnosed ACL ruptures and 100 healthy controls between 18 and 28 years of age without history of 
ligament or tendon injuries were recruited for the study. All participants were genotyped for the MMP-3 polymorphism (-1612 5A/6A). Statistical analyses of genotype frequencies between patients and healthy controls were performed by the chi-square test. A significant difference was found between ACL rupture subgroups in terms of genotype association $(5 \mathrm{~A}+(5 \mathrm{~A} / 5 \mathrm{~A}, 5 \mathrm{~A} / 6 \mathrm{~A}): 37.5 \%$ in contact sports $v s 20 \%$ in non-contact sports; $\mathrm{P}=0.02)$. In allelic association, there were significant differences (6A: $81.2 \%$ in contact sports vs $89.1 \%$ in non-contact sports, $5 \mathrm{~A}: 18.8 \%$ in contact sports $v_{s} 10.9 \%$ in non-contact sports, $\mathrm{P}=0.01$ ). The $5 \mathrm{~A}+$ genotype of MMP-3 was represented in ACL ruptures in contact sport participants. We propose that this sequence variant is a specific genetic element that should be included in a multifactorial model to understand the etiologies and risk factors for ACL rupture.

Key words: Anterior cruciate ligament ruptures; Matrix metalloproteinase-3; Single nucleotide polymorphism

\section{INTRODUCTION}

Anterior cruciate ligament (ACL) injury is one of the most common injuries in a sporting population (Brooks et al., 2005). Athletes are involved with sudden deceleration of the body from jumping and forward running while the knee is in a shallow flexion angle. Sudden change in particular direction, such as in soccer, rugby and football, contributes to a higher risk of rupturing the ACL (Marshall et al., 2007). ACL rupture risk in athletes was ten times greater than that in the general population (de Loes et al., 2000). Severe ACL injury leads to the development of knee osteoarthritis (Deacon et al., 1997).

The ACL consists of two major fiber bundles, namely the anteromedial (AM) and posterolateral (PL) bundles, which display a shape comparable to a vertically arranged semicircle. The bony attachment is located at the posterior part of the inner surface of the lateral femoral condyle (Duthon et al., 2006). It is a key structure in the knee joint, as it resists anterior tibial translation and rotational loads. In the physical examination of the ACL injury, the Lachman test would be effective in ruling out an ACL rupture in a setting involving a wide range of knee injuries, while the pivot shift test may be effective in diagnosing an ACL (Stevens and Dragoo, 2006).

Even though ACL tears occur frequently, healing never occurs and reconstructive surgery may be the only alternative for patients who develop symptoms of knee instability. Reconstruction is aimed at restoring the kinematics and stability of the injured knee. Individuals choosing to forego surgery after a severe ACL injury may be restricted to a sedentary lifestyle and increased morbidity. Approximately $80 \%$ of patients will develop radiographic signs, and $40-50 \%$ will develop overt symptoms of osteoarthritis after an ACL tear if the injury is not repaired (Deacon et al., 1997).

Although intrinsic and extrinsic factors for ACL ruptures have been identified, the exact etiology of this injury is not yet fully understood. Many studies have suggested that genetic elements should be considered as an intrinsic risk factor for ACL rupture. It was previously shown that individuals who had blood relative (family) history of ACL rupture exhibited 2-fold higher risk for ACL rupture (Flynn et al., 2005). Although no specific genes have been 
identified, a genetic component may play a part in ACL ruptures. Moreover, IL-6, matrix metalloproteinases (MMP)-1 and MMP-3 in synovial fluid have been evaluated after ligament injury (Higuchi et al., 2006).

MMPs are members of the zine-endopeptidases (Nagase et al., 2006). MMPs can be divided into five groups; collagenases (MMP-1, MMP-8 and MMP-13), gelatinases (MMP-2 and MMP-9), stromelysins (MMP-3, MMP-7 and MMP-10), matrilysins, and membrane-type MMPs (MT-MMPs). They have the ability to degrade extracellular matrix. Most MMPs are synthesized by fibroblasts. MMP-3 (stomelysin-1) is a member of the MMPs responsible for the degradation of collagen and activation of proMMP-1. In normal tissues, MMP expression is low, but in extracellular matrix remodeling, altered expression of MMP is required. MMP-3 is involved in many biological functions, including extracellular matrix remodeling and degradation, cell proliferation and angiogenesis (Chakraborti et al., 2003).

The MMP-3 gene is located on the long arm of chromosome 11 (11q22.3), and the level of expression is influenced by single nucleotide polymorphisms (SNPs) in the promoter region. One allele has five adenosines and other allele has six adenosines. The -1612 position in the promoter region has an insertion/deletion polymorphism $(5 \mathrm{~A} / 6 \mathrm{~A})$ leading to a difference in expression of MMP-3 proteins. A previous study showed that the $5 \mathrm{~A}$ allele had a 2 -fold higher activity than the $6 \mathrm{~A}$ allele, and therefore, the $5 \mathrm{~A}$ allele results in higher gene expression compared to the $6 \mathrm{~A}$ allele. In addition, the $5 \mathrm{~A} / 6 \mathrm{~A}$ polymorphism in the promoter of MMP-3 gene has been shown to be associated with lumbar disc degeneration (Takahashi et al., 2001; Yuan et al., 2010), myocardial infarction (Samnegard et al., 2005), rheumatoid arthritis (Mattey et al., 2004), and periodontitis (Astolfi et al., 2006).

The purpose of this study was to determine the association of the $-16125 \mathrm{~A} / 6 \mathrm{~A}$ polymorphism in the MMP-3 gene in ACL patients and healthy controls.

\section{MATERIAL AND METHODS}

\section{Study population}

This study was approved by the Institutional Ethics Committee on Human Research. All the participants were informed of the study's purposes, and written informed consent was obtained prior to participation in this study. Each participant provided personal details and family history of ligament injury, and filled out a sport participation questionnaire. Sport participation was classified into contact sports and non-contact sports.

A total of 186 participants were included in the present study. Eighty-six participants with surgically diagnosed ACL ruptures were recruited from the Sport Medicine Clinics, King Chulalongkorn Memorial Hospital. In addition, 100 apparently healthy controls who did not have a history of ligament injury participated in similar sports as the ACL patients. The ACL patients and controls were gender matched.

\section{Isolation of genomic DNA}

Genomic DNA was extracted from blood samples using Illustra Blood GenomicPrep Mini Spin kit (GE Healthcare, UK). The extracted DNA was quantified and checked for purity by Nanodrop (Thermo Scientific, USA). 


\section{Genotype of the MMP-3 polymorphism}

The MMP-3 genotype was determined using the polymerase chain reaction-restriction fragment length polymorphism (PCR-RFLP) assay. The PCR primers for amplifying the MMP-3 promoter were: forward primer 5'-GATTACAGACATGGGTCACG-3' and reverse primer 5'-TTTCAATCAGGACAAGACGAAGTTT-3' (Dunleavey et al., 2000). A mismatch close to the 3'-end of the reverse primer was made to create a restriction site in the case of the 5A allele by XmnI (New England BioLabs, UK). PCR was performed in a $20-\mu \mathrm{L}$ volume containing $5 \mu \mathrm{L}$ genomic DNA template, $2 \mu \mathrm{L}$ 10X Taq buffer, $2.5 \mathrm{mM} \mathrm{MgCl}_{2}, 2 \mathrm{mM}$ dNTPs, $200 \mathrm{nmol}$ forward and reverse primer, and $4 \mathrm{U}$ Taq DNA polymerase (Fermentas Inc., USA). The PCR protocol was $95^{\circ} \mathrm{C}$ denaturation followed by 35 cycles: denaturation at $95^{\circ} \mathrm{C}$ for 45 $\mathrm{s}$, annealing at $53^{\circ} \mathrm{C}$ for $45 \mathrm{~s}$ and elongation at $72^{\circ} \mathrm{C}$ for $30 \mathrm{~s}$, ending with an additional elongation step of $5 \mathrm{~min}$ at $72^{\circ} \mathrm{C}$. For a negative control, each PCR contained distilled water instead of DNA template in the reaction mixture.

\section{Restriction enzyme (XmnI) digestion of the MMP-3 gene}

A 5- $\mu \mathrm{L}$ aliquot of PCR product was digested at $37^{\circ} \mathrm{C}$ for $3 \mathrm{~h}$ in $10 \mu \mathrm{L}$ reaction mixture containing of $4 \mathrm{U} X m n \mathrm{I}$ and $1 \mathrm{X}$ NEB buffer 4 and $3.8 \mu \mathrm{L}$ distilled water. After digestion, the reaction mixtures were analyzed by electrophoresis on a $12 \%$ polyacrylamide gel stained with ethidium bromide and visualized using ultraviolet transillumination. On electrophoresis, the 5A bands were represented by 97 and $23 \mathrm{bp}$, the $6 \mathrm{~A}$ bands were represented by $120 \mathrm{bp}$, whereas the heterozygote displayed a combination of both sets of bands (120, 93 and $23 \mathrm{bp}$ ).

\section{Statistical analysis}

The chi-square test ( $\chi^{2}$ test) was used to calculate the difference in genotypic distribution of the MMP-3 polymorphism between the ACL and control groups. A P value $<0.05$ was considered to be statistically significant. For each parameter, patients with another genotype were analyzed by odds ratios (OR) and 95\% confidence intervals (CI). Distributions of MMP-3 polymorphisms in ACL patients and controls were analyzed to see if they are in Hardy-Weinberg equilibrium. The statistical analysis was performed by the SPSS 16.0 software package (SPSS Inc., USA).

\section{RESULTS}

\section{Patient characteristics}

The demographic data of ACL patients and healthy controls are presented in Table 1. There were significant differences in age and body mass index (BMI) between these two groups. Particularly, the proportion of those in their 20s was greater in the control group, whereas the proportion of those in their 30s was higher to some extent in the patient group. The BMI of ACL patients was significantly higher than that of controls $(\mathrm{P}=0.003)$. The ACL patients and controls were gender matched. In ACL patients, there was no statistically significant difference between the patients with and without contact sports (Table 2). 
Table 1. General characteristics of the control group and the anterior cruciate ligament rupture (ACL) group.

\begin{tabular}{lcrr}
\hline & Controls $(\mathrm{N}=100)$ & ACL $(\mathrm{N}=86)$ & P value \\
\hline Age $($ years $)$ & $23.2 \pm 5.3$ & $30.6 \pm 8.4$ & $<0.001$ \\
Height $(\mathrm{cm})$ & $167.6 \pm 7.9$ & $171.6 \pm 6.9$ & $<0.001$ \\
Weight $(\mathrm{kg})$ & $63.8 \pm 11.5$ & $70.7 \pm 9.8$ & $<0.001$ \\
BMI $\left(\mathrm{kg} / \mathrm{m}^{2}\right)$ & $22.2 \pm 4.7$ & $23.9 \pm 2.8$ & 0.003 \\
\hline
\end{tabular}

\begin{tabular}{lccr} 
Table 2. General characteristics of the anterior cruciate ligament rupture (ACL) subgroup. & \\
\hline & \multicolumn{2}{c}{ ACL subgroups, based on mechanism of injury } & P value \\
\cline { 2 - 4 } & Contact sports ( $=40)$ & Non-contact sports (N=46) & 0.07 \\
\hline Age (years) & $29.2 \pm 6.9$ & $31.9 \pm 9.5$ & 0.24 \\
Height $(\mathrm{cm})$ & $172.6 \pm 6.9$ & $170.9 \pm 7.0$ & 0.90 \\
Weight $(\mathrm{kg})$ & $70.5 \pm 11.5$ & $70.8 \pm 8.5$ & 0.32 \\
BMI $\left(\mathrm{kg} / \mathrm{m}^{2}\right)$ & $23.6 \pm 3.3$ & $24.2 \pm 2.4$ & \\
\hline
\end{tabular}

\section{MMP-3 genotype frequencies}

Table 3 illustrates genotype and allele frequencies. These data of allele frequencies conform to Hardy-Weinberg equilibrium and will remain unchanged generation after generation in a hypothetical situation. The frequency of the $5 \mathrm{~A}$ allele was 0.11 in the control group and 0.14 in the ACL group. This is similar to that in a population in Asia reported by Takahashi et al. (2001) and Liu et al. (2010), although different from those in South Africa reported by Posthumus et al. (2009).

There were no significant differences in the distribution of genotype $5 \mathrm{~A}+$ between ACL and control group $(\mathrm{P}=0.38)$, and of genotype 5A- between ACL and control group ( $\mathrm{P}$ $=0.62)$, or allele between ACL and control group $(\mathrm{P}=0.39)$ in the frequencies of the MMP-3 (-1612) promoter polymorphism (Table 3). However, in ACL patients, there were significant differences in genotype $5 \mathrm{~A}+$ between the ACL patients with contact sport and without contact sport $(\mathrm{P}=0.02, \mathrm{OR}=2.25,95 \% \mathrm{CI}=1.18-4.25)($ Tables 3 and 4$)$.

\begin{tabular}{|c|c|c|c|c|c|c|}
\hline & \multirow[t]{2}{*}{ Controls $(\mathrm{N}=100)$} & \multirow[t]{2}{*}{ ACL $(\mathrm{N}=86)$} & \multirow[t]{2}{*}{$\mathrm{P}$ value } & \multicolumn{2}{|c|}{ ACL subgroups, based on mechanism of injury } & \multirow[t]{2}{*}{$P$ value } \\
\hline & & & & Contact sports $(\mathrm{N}=40)$ & Non-contact sports $(\mathrm{N}=46)$ & \\
\hline $\begin{array}{l}5 \mathrm{~A}+\text { genotype } \\
(5 \mathrm{~A} / 5 \mathrm{~A}+5 \mathrm{~A} / 6 \mathrm{~A})\end{array}$ & $1+20(21.0 \%)$ & $1+22(26.7 \%)$ & 0.38 & $15(37.5 \%)$ & $1+8(20.0 \%)$ & 0.02 \\
\hline $5 \mathrm{~A}-$ genotype $(6 \mathrm{~A} / 6 \mathrm{~A})$ & $79(79.0 \%)$ & $63(73.3 \%)$ & 0.62 & $25(62.5 \%)$ & $37(80.0 \%)$ & 0.18 \\
\hline $5 \mathrm{~A}$ allele frequency & 0.11 & 0.14 & & 0.19 & 0.10 & \\
\hline $5 \mathrm{~A}$ allele $(\%)$ & $11.0 \%$ & $13.9 \%$ & 0.39 & $18.8 \%$ & $10.9 \%$ & 0.01 \\
\hline 6 A allele $(\%)$ & $89.0 \%$ & $86.1 \%$ & & $81.2 \%$ & $89.1 \%$ & \\
\hline
\end{tabular}

Table 4. Risk estimation of the MMP-3 gene in anterior cruciate ligament rupture (ACL) patients.

\begin{tabular}{lccc}
\hline Genotype & Odds ratio & $95 \%$ CI & P value \\
\hline $5 \mathrm{~A}+$ & 1.39 & $0.72-2.67$ & 0.41 \\
$5 \mathrm{~A}-$ & 0.72 & $0.37-1.38$ & 0.40 \\
$5 \mathrm{~A}+$ with contact sports & 2.25 & $1.18-4.25$ & 0.02 \\
\hline
\end{tabular}




\section{DISCUSSION}

The main finding of this study was that the $5 \mathrm{~A}+$ genotype of the MMP-3 promoter polymorphism was significantly different among contact sport patients with ACL injuries. In addition, the observation from this study suggests that the MMP-3 5A+ genotype of the -1612 promoter polymorphism was associated with susceptibility to ACL rupture in the contact sport patients.

Our study of an under-representation of the $5 \mathrm{~A}+$ genotype in ACL in contact sport patients was different than in ACL in non-contact sport patients. Furthermore, the genotype distributions in the control group were similar to those in previous studies of Asian participants (Lu et al., 2007; Liu et al., 2010). Furthermore, the common mechanism of ACL rupture occurred with non-contact sports more than with contact sports. Non-contact sport injury occurs on its own movement. On the other hand, contact sport injury occurs when there is contact with other athletes or objects. It may be hypothesized that the intrinsic factors (genetic elements) combined with the extrinsic risk factors (external forces) may play a potential role in ACL ruptures.

Biochemically, the mechanisms of ligament injuries are poorly elucidated. These injuries are considered to be multifactorial disorders. The risk factors are divided into intrinsic and extrinsic factors. However, ACL injuries may be enhanced by breakdown of the matrix, since the main components of the ACL are collagen fibrils (Yougu, 2004). Thus, they could be degraded by a class of proteoglycan enzymes such as MMPs that have been demonstrated to play a role in tissue degradation (Collins and Raleigh, 2009). In addition, MMP synthesis and functions are regulated by transcriptional activation and post-transcriptional processing and inhibited by endogenous inhibitors, called tissue inhibitors of metalloproteinases (TIMPs).

MMP-3 is a potent proteoglycan-degrading enzyme that plays an important role in degrading collagen and breaking down other structural components such as proteoglycan, laminin, gelatin, and fibronectin (Matrisian, 1990). MMP-3 also indirectly affects the degradation of the extracellular matrix by activating other latent MMPs. The common $5 \mathrm{~A} / 6 \mathrm{~A}$ polymorphism in the promoter of MMP-3 has an influence on the level of MMP-3 gene expression in vivo. The equilibrium between MMPs and TIMPs regulates extracellular matrix degradation. The $5 \mathrm{~A}$ allele was associated with higher transcriptional activity whereas the $6 \mathrm{~A}$ allele had a lower level of MMP-3 gene expression (Ye, 2000).

A limitation of our study is that the exposure to extrinsic risk factors (high-risk sports such as sports involving cutting, pivoting and landing) could not be well documented in the control group. Furthermore, the BMI of the ACL patients was significantly higher than that of controls. These differences are non-genetic factors but they may be variables in determining injury risk of ACL ruptures.

The results of this study support the hypothesis that genetic factors are associated with the risk factors of ACL ruptures. However, ACL ruptures represent a multifactorial disorder and the etiology of ACL rupture remains unclear, because of the complex interaction between the intrinsic and extrinsic risk factors. Therefore, this study may be another model for better understanding the etiology and risk factors of ACL ruptures.

\section{CONCLUSION}

This study found that the occurrence of MMP-3 5A+ genotype of the -1612 promoter 
polymorphism was significant in ACL ruptures with contact sport compared with healthy controls in an independent population. This polymorphism could be a specific genetic element to be included in multifactorial models to understand the etiology and risk factors of ACL injury.

\section{ACKNOWLEDGMENTS}

Research supported by the CU Graduate School Thesis Grant, the Ratchadapiseksompotch Fund, Chulalongkorn University, the Thailand Research Fund, Commission on Higher Education, and the National Research Council of Thailand. The authors also thank Professor Kiat Ruxrungtham, the HIV-NAT Research Collaboration, the Thai Red Cross AIDS Research Centre, the Core Facility of the Department of Biochemistry, and the Chulalongkorn Medical Research Center (ChulaMRC) for kindly providing facilities. Research was also supported in part by the National Research University Project of CHE and the Ratchadaphiseksomphot Endowment Fund (\#HR1155A).

\section{REFERENCES}

Astolfi CM, Shinohara AL, da Silva RA, Santos MC, et al. (2006). Genetic polymorphisms in the MMP-1 and MMP-3 gene may contribute to chronic periodontitis in a Brazilian population. J. Clin. Periodontol. 33: 699-703.

Brooks JH, Fuller CW, Kemp SP and Reddin DB (2005). Epidemiology of injuries in English professional rugby union: part 1 match injuries. Br. J. Sports Med. 39: 757-766.

Chakraborti S, Mandal M, Das S, Mandal A, et al. (2003). Regulation of matrix metalloproteinases: an overview. Mol. Cell. Biochem. 253: 269-285.

Collins M and Raleigh SM (2009). Genetic risk factors for musculoskeletal soft tissue injuries. Med. Sport Sci. 54: 136149.

de Loes M, Dahlstedt LJ and Thomee R (2000). A 7-year study on risks and costs of knee injuries in male and female youth participants in 12 sports. Scand. J. Med. Sci. Sports 10: 90-97.

Deacon A, Bennell K, Kiss ZS, Crossley K, et al. (1997). Osteoarthritis of the knee in retired, elite Australian rules footballers. Med. J. Aust. 166: 187-190.

Dunleavey L, Beyzade S and Ye S (2000). Rapid genotype analysis of the stromelysin gene 5A/6A polymorphism. Atherosclerosis 151: 587-589.

Duthon VB, Barea C, Abrassart S, Fasel JH, et al. (2006). Anatomy of the anterior cruciate ligament. Knee Surg. Sports Traumatol. Arthrosc. 14: 204-213.

Flynn RK, Pedersen CL, Birmingham TB, Kirkley A, et al. (2005). The familial predisposition toward tearing the anterior cruciate ligament: a case control study. Am. J. Sports Med. 33: 23-28.

Higuchi H, Shirakura K, Kimura M, Terauchi M, et al. (2006). Changes in biochemical parameters after anterior cruciate ligament injury. Int. Orthop. 30: 43-47.

Liu Z, Tang NL, Cao XB, Liu WJ, et al. (2010). Lack of association between the promoter polymorphisms of MMP-3 and IL-6 genes and adolescent idiopathic scoliosis: a case-control study in a Chinese Han population. Spine 35: 17011705.

Lu Z, Cao Y, Wang Y, Zhang Q, et al. (2007). Polymorphisms in the matrix metalloproteinase-1, 3, and 9 promoters and susceptibility to adult astrocytoma in northern China. J. Neurooncol. 85: 65-73.

Marshall SW, Padua D and McGrath M (2007). Incidence of ACL injuries. Hum. Kinet. 5-30.

Matrisian LM (1990). Metalloproteinases and their inhibitors in matrix remodeling. Trends Genet. 6: 121-125.

Mattey DL, Nixon NB, Dawes PT, Ollier WE, et al. (2004). Association of matrix metalloproteinase 3 promoter genotype with disease outcome in rheumatoid arthritis. Genes Immun. 5: 147-149.

Nagase H, Visse R and Murphy G (2006). Structure and function of matrix metalloproteinases and TIMPs. Cardiovasc. Res. 69: 562-573.

Posthumus M, September AV, Keegan M, O’Cuinneagain D, et al. (2009). Genetic risk factors for anterior cruciate ligament ruptures: COL1A1 gene variant. Br. J. Sports Med. 43: 352-356.

Samnegard A, Silveira A, Lundman P, Boquist S, et al. (2005). Serum matrix metalloproteinase-3 concentration is influenced by MMP-3 -1612 5A/6A promoter genotype and associated with myocardial infarction. J. Intern. Med. 
258: 411-419.

Stevens KJ and Dragoo JL (2006). Anterior cruciate ligament tears and associated injuries. Top. Magn. Reson. Imaging 17: 347-362.

Takahashi M, Haro H, Wakabayashi Y, Kawauchi T, et al. (2001). The association of degeneration of the intervertebral disc with $5 \mathrm{a} / 6 \mathrm{6}$ polymorphism in the promoter of the human matrix metalloproteinase-3 gene. J. Bone Joint Surg. $\mathrm{Br}$. 83: 491-495.

Ye S (2000). Polymorphism in matrix metalloproteinase gene promoters: implication in regulation of gene expression and susceptibility of various diseases. Matrix Biol. 19: 623-629.

Yougu H (2004). Hernia of intervertebral discs in Chinese. People's Medical Publishing House.

Yuan HY, Tang Y, Liang YX, Lei L, et al. (2010). Matrix metalloproteinase-3 and vitamin d receptor genetic polymorphisms, and their interactions with occupational exposure in lumbar disc degeneration. J. Occup. Health 52: $23-30$. 